\title{
ANÁLISIS DEL CONTENIDO POLÍNICO DE LA ATMÓSFERA DE HUELVA (1989-1992)
}

\author{
Francisco José GONZÁLEZ MINERO y Pilar CANDAU
}

RESUMEN. Análisis del contenido polínico de la atmósfera de Huelva (1989-1992). En este trabajo se presenta el contenido polínico de la atmósfera de Huelva encontrado tras cuatro años de muestreo (19891992) utilizando un captador polínico tipo Cour. Durante este periodo se han identificado 82 tipos polínicos diferentes, de los que 28 tienen una representación superior al $0.01 \%$ en el polen total recogido. El polen de origen arbóreo (Quercus, Pinaceae, Olea, Myrtaceae, Cupressaceae, Casuarina, Fraxinus, Alnus, Castanea, etc.), con un 64\%, predomina sobre el de origen herbáceo (34\%) (Poaceae, Amaranthaceael Chenopodiaceae, Urticaceae, Plantago, Rumex, Compositae, etc.), el $2 \%$ restante es arbustivo, siendo Ericaceae el más abundante. Desde el punto de vista cuantitativo los tipos polínicos aparecen ordenados como sigue: Poaceae (18.08\%), Quercus (14.75\%), Pinaceae (13.49\%), Olea (11.79\%), Myrtaceae (11.41\%), Amaranthaceae/ Chenopodiaceae (11.03\%), Urticaceae (8.07\%) y Cupressaceae (5.13\%). A lo largo del año existen varios periodos de máxima emisión polínica: febrero (Cupressaceae), final de invierno (Fraxinus, Platanus y Urticaceae), mediados de abril (Quercus y Pinaceae), mediados de mayo (Poaceae, Olea, Plantago y Rumex), final de junio (Myrtaceae), final de agosto (Amaranthaceael Chenopodiaceae) y octubre (Casuarina). Por último, aparecen cantidades bajas de polen entomófilo o mixto: Castanea, Compositae, Cruciferae, Boraginaceae, Ericaceae, Helianthus, Leguminosae y Umbelliferae.

Palabras clave.Aeropalinología, método Cour, polen anemófilo, Huelva, España.

ABSTRACT. Analysis of the pollen content in the Huelva atmosphere (1989-1992). The airborne pollen of Huelva city is presented. It has been established after four consecutive years of sampling (1989-1992) using a Cour's trap. We identifyed 82 pollen types in out of which 28 were presented over $0.01 \%$ in the total amount. Pollen belonging to tree species (Quercus, Pinaceae, Olea, Myrtaceae, Cupressaceae, Casuarina, Fraxinus, Alnus, Castanea, etc.), a meant $64 \%$ and predominated over that of weed species (34\%) (Poaceae, Amaranthaceael Chenopodiaceae, Urticaceae, Plantago, Rumex, Compositae), and shurb species (2\%) (Ericaceae is the most frequent in this group). From a quantitative point of view, the main pollinic types were as follows: Poaceae (18.08\%), Quercus (14.75\%), Pinaceae (13.49\%), Olea (11.79\%), Myrtaceae $(11.41 \%)$, Amaranthaceael Chenopodiaceae $(11.03 \%)$, Urticaceae $(8.07 \%)$ and Cupressaceae $(5.13 \%)$. Throughout the year there were five periods of maximun emission of pollen: The end of winter (Fraxinus, Cupressaceae, Platanus and Urticaceae), middle of April (Quercus and Pinaceae), middle of May (Poaceae, Olea, Plantago and Rumex), end of June (Myrtaceae), end of August (Amaranthaceael Chenopodiaceae) and October (Casuarina). Additionally, small amounts of totally or partially antomophylous pollen, were found: Castanea, Compositae, Cruciferae, Boraginaceae, Ericaceae, Helianthus, Leguminosae and Umbelliferae.

Key words. Aeropalinology, anemophylous pollen, Cour's method, Huelva, Spain. 


\section{INTRODUCCIÓN}

El estudio del contenido polínico de un área geográfica determinada posee gran aplicación en los campos de la medicina (Hurtado \& Alson, 1990), agrícola (Cour \& Van Campo, 1980) y medioambiental (Keynan et al., 1991), de ahí que trabajos de este tipo se hayan realizado o estén desarrollándose en numerosas ciudades de países industrializados.

Bajo esta perspectiva se exponen a continuación los resultados del muestreo aeropalinológico realizado en la ciudad de Huelva entre 1989 y 1992, que amplian los estudios parciales llevados a cabo por los autores (Candau y González Minero, 1992, Candau et al., 1993) y complementan los trabajos realizados en otras ciudades de Andalucía: Cádiz (Díaz Rubio y Barrios Gutiérrez, 1942), Córdoba (Galán, 1987), Granada (Fernández et al., 1990), Sevilla (González Romano et al., 1992, 1993) y Málaga (Cabezudo et al., 1994).

La ciudad de Huelva está situdada en la costa atlántica al SO de España $\left(37^{\circ} 16^{\prime} \mathrm{N}, 6^{\circ}\right.$ $\left.16^{\circ} \mathrm{O}\right)$. Su clima es mediterráneo marítimo con $18^{\circ} \mathrm{C}$ de temperatura media anual y $470 \mathrm{~mm} \mathrm{de}$ lluvia (Almarza Mata, 1984).

La flora que potencialmente influye en el espectro polínico de la ciudad se puede dividir en autóctona e introducida. En el primer grupo destacan: elementos característicos del bosque mediterráneo, encinar (Quercus rotundifolia) y alcornocal (Quercus suber), sustituidos respectivamente por jarales (Cistus sp.) y brezales (Erica sp.); representantes de las familias Cupressaceae (Juniperus oxycedrus y Jupinerus phoenicea) y Compositae presentes en dunas costeras (Artemisia caerulescens y Artemisia crithmifolia); comunidades palustres (Typhaceae entre otras); comunidades de riberas (Alnus y Fraxinus); especies halófitas de las familias Chenopodiaceae, Poaceae y Cyperaceae, adaptadas a las condiciones de alta salinidad de las marismas próximas a la ciudad; elementos de comunidades nitrófiloruderales localizadas en un entorno urbano en el que baldíos y escombreras son abundantes (Amaranthaceae, Chenopodiaceae, Mercurialis, Plantago, Rumex, Poaceae, Umbelliferae y Urticaceae).

El segundo grupo está constituido por: representantes forestales fruto de extensas repoblaciones pasadas y recientes (Castanea sativa, Pinaceae y Myrtaceae); cultivos arbóreos (30000 has. de olivar y 18000 Has. de viñedo) y extensiones variables de cultivos herbáceos (cereales, leguminosas y girasol); por último, elementos ornamentales de parques y jardines, relativamente escasos en Huelva durante este periodo (Acacia, Casuarina, Cupressaceae, Fraxinus, Ligustrum, Moraceae, Palmae y Platanus).

\section{MATERIAL Y MÉTODOS}

Se han realizado muestreos semanales del aire de Huelva durante los años 1989-1992 empleando un captador Cour situado a $15 \mathrm{de}$ metros de altura en el centro de la ciudad. La metodología utilizada en el procesado de las muestras ha sido la descrita por Cour en 1974, mediante la cual se obtienen preparaciones de polen acetolizado y coloreado en un medio semifluido, condiciones que facilitan su identificación.

La elección de los tipos polínicos que presentamos se ha hecho atendiendo a su importancia cuantitativa en el muestreo. Los tipos polínicos establecidos coinciden en su mayoría con los descritos por Valdés et al., (1987). Los límites taxonómicos son muy elásticos, los tipos pueden incluir polen de una especie, un género y una familia. Esta heterogeneidad viene impuesta en parte por las limitaciones ópticas a la hora de la identificación de los mismos.

Para los tipos polínicos más importantes se especifica, los granos $/ \mathrm{m}^{3}$ de polen anual 
recogido, la semana de máxima emisión polínica (SMEP), las semanas que comprenden su periodo de polinización principal (PPP) (Muellenders et al., 1972) y su porcentaje de participación polínica $(\mathrm{P})$ en el polen total recogido al año.

Así mismo se ha construido en cada caso una figura en la que se visualiza la curva polínica anual o variación anual de las concentraciones polínicas semanales, en la que en el eje de abcisas se representan semanas y meses y en el eje de ordenadas las concentraciones polínicas expresadas en granos $/ \mathrm{m}^{3}$ (en cada tipo se usa la escala más apropiada para su comprensión gráfica). Todos los valores señalados son medias cuatrianuales realizadas a partir de las observaciones llevadas a cabo entre 1989 y 1992.

\begin{tabular}{lrrrr}
\hline TIPO & Polen Total & SMEP & PPP & P $(\%)$ \\
\hline Alnus & 42.18 & 10 & $3-10$ & 0.49 \\
Amar/Chen & 957.67 & 35 & $21-37$ & 11.03 \\
Artemisia & 10.77 & 36 & $34-51$ & 0.12 \\
Boraginacae & 23.37 & 21 & $13-26$ & 0.27 \\
C.equinuladas & 145.93 & 21 & $15-31$ & 1.68 \\
C.fenestradas & 21.85 & 18 & $16-29$ & 0.25 \\
Castanea & 2.83 & 27 & $19-28$ & 0.03 \\
Casuarina & 170.43 & 42 & $37-49$ & 1.96 \\
Cruciferae & 40.79 & 13 & $6-40$ & 0.47 \\
Cupressaceae & 446.16 & 8 & $4-39$ & 5.13 \\
Cyperaceae & 24.97 & 26 & $15-26$ & 1.19 \\
Ericaceae & 103.67 & 21 & $13-22$ & 1.16 \\
Fraxinus & 101.20 & 13 & $3-18$ & 0.55 \\
Leguminosae & 47.84 & 20 & $13-21$ & 0.76 \\
Mercurialis & 66.32 & 5 & $3-20$ & 0.26 \\
Moraceae & 23.06 & 10 & $6-18$ & 11.41 \\
Myrtaceae & 991.74 & 26 & $25-29$ & 11.79 \\
Olea & 1024.13 & 21 & $18-22$ & 13.43 \\
Palmae & 124.57 & 21 & $10-32$ & 3.08 \\
Pinaceae & 1172.40 & 17 & $10-21$ & 0.87 \\
Plantago & 267.15 & 21 & $13-25$ & 18.08 \\
Platanus & 76.29 & 13 & $11-13$ & 14.75 \\
Poaceae & 1570.91 & 20 & $13-28$ & 1.78 \\
Quercus & 1281.21 & 15 & $13-21$ & 0.33 \\
Rumex & 154.54 & 21 & $8-21$ & 0.28 \\
Typhaceae & 28.5 & 21 & $10-33$ & 8.07 \\
Umbelliferae & 24.21 & 21 & $19-30$ & \\
Urticaceae & 701.00 & 13 & $5-20$ & \\
\hline
\end{tabular}

Tabla 1. Resumen de los datos aeropalinológicos de los tipos más importantes del espectro polínico de Huelva. Valores obtenidos a partir de medias cuatrianuales (1989-1992). Incluye polen total recogido al año, semana de máxima emsión polínica (SMEP), periodo de polinización principal (PPP) y porcentaje de participación en el espectro polínico (\%). Summary of the aeropalynology data of the most important pollen types found in the Huelva pollen spectrum. The achivied outcome are based upon four years period averages (1989-1992). Been here in considered the total quantity of pollen trapped in one years basis, the week of highest pollinic emission (SEMP), the main pollination period (PPP) and a percentage of participation in the pollen spectrum $(P)$. 


\section{RESULTADOS}

Durante los cuatro años de muestreo se han detectado 82 tipos polínicos en la atmósfera de Huelva, de los que 28 tienen una representación en el espectro superior al $0.01 \%$, dichos tipos se recogen ordenados alfabéticamente en la tabla 1, criterio que seguimos para presentar las curvas aeropalinológicas (figs. 1-28) con el fin de visualizar la variación del contenido polínico muestreado a lo largo del año. En el anexo se recogen los taxones que integran los tipos polínicos objeto de este estudio.

La vegetación descrita anteriormente se refleja en el contenido polínico del aire de la ciudad, los tipos más abundantes son: Poaceae (18.08\%), Quercus (14.75\%), Pinaceae (13.49\%), Olea (11.79\%), Myrtaceae (11.41\%), Amaranthaceael Chenopodiaceae (11.03\%), Urticaceae $(8.07 \%)$ y Cupressaceae $(5.13 \%)$. En el otro extremo, con una representación inferior al $0.60 \%$ cada uno, están Alnus, Artemisia, Cyperaceae, Moraceae, Typhaceae y tipos de procedencia entomófila (Castanea, Compuestas fenestradas (Lactucoideae), Cruciferae, Boraginaceae, Leguminosae y Umbelliferae) (tab. 1).

Según la permanencia de los tipos en el espectro (tab. 1), se pueden establecer tres grupos:

- Tipos con periodo de polinización principal corto, inferior a 10 semanas de duración. La curva polínica es homogénea, caracterizada por la presencia de uno o dos picos. Son tipos que incluyen por lo general, una o pocas especies que florecen al mismo tiempo: Alnus glutinosa, Platanus hybrida, Olea europaea, Castanea sativa, Leguminosae, Quercus y Myrtaceae.

- Tipos con periodo de polinización principal medio, entre 9 y 14 semanas de duración: Moraceae, Pinaceae, Ericaceae, Plantago, Boraginaceae, Rumex, Cyperaceae, Compuestas fenestradas (Lactucoideae),

\section{Umbelliferae y Casuarina.}

- Tipos con periodo de polinización principal largo, igual o superior a 15 semanas: Fraxinus, Mercurialis, Cupressaceae, Urticaceae, Cruciferae, Palmae, Typhaceae, Compuestas equinuladas (Asteroideae), Amaranthaceae/Chenopodiaceae y Artemisia.

Las curvas polínicas de los dos últimos grupos presentan subidas y bajadas de las concentraciones adoptando un perfil de dientes de sierra (véanse figuras), debido por una parte a que son tipos que se corresponden con familias o géneros integrados por numerosos taxones (anexo) con floración gradual y fenología particular (Poaceae, Amaranthaceael Chenopodiaceae, Compositae, Cupressaceae, Urticaceae, etc.) y por otra a que son tipos de los que se recogen intermitentemente pequeñas cantidades de polen (Cruciferae, Palmae, Moraceae, Typhaceae, Artemisia, etc.) (véanse figuras).

Considerando la diversidad polínica mensual y las concentraciones máximas alcanzadas, el contenido polínico se puede globalizar de la siguiente forma (fig. 1).

De enero a marzo domina en el aire el polen de Alnus, Fraxinus, Cupressaceae, Urticaceae y Mercurialis. En la semana 8 (febrero) se alcanza la máxima concentración de Cupresaaceae (60 granos $/ \mathrm{m}^{3}$ ), en la 10 (marzo) las máximas concentraciones de Moraceae (2.5 granos $/ \mathrm{m}^{3}$ ) y Alnus (16 granos/ $\mathrm{m}^{3}$ ) y en la 13 (marzo) las máximas concentraciones de Fraxinus (12 granos $/ \mathrm{m}^{3}$ ), Urticaceae $\left(60 \mathrm{granos} / \mathrm{m}^{3}\right)$ y Platanus (30 granos $\left./ \mathrm{m}^{3}\right)$.

En abril se recogen las concentraciones más altas de Quercus (250 granos $/ \mathrm{m}^{3}$ en la semana 15 ) y Pinaceae (300 granos $/ \mathrm{m}^{3}$ en la semana 17).

Mayo es el mes de mayor diversidad polínica en el aire y en el que se registran concentraciones más altas de polen. En la semana 18 se alcanza la mayor emisión polínica de Compuestas fenestradas (Lactucoideae) 

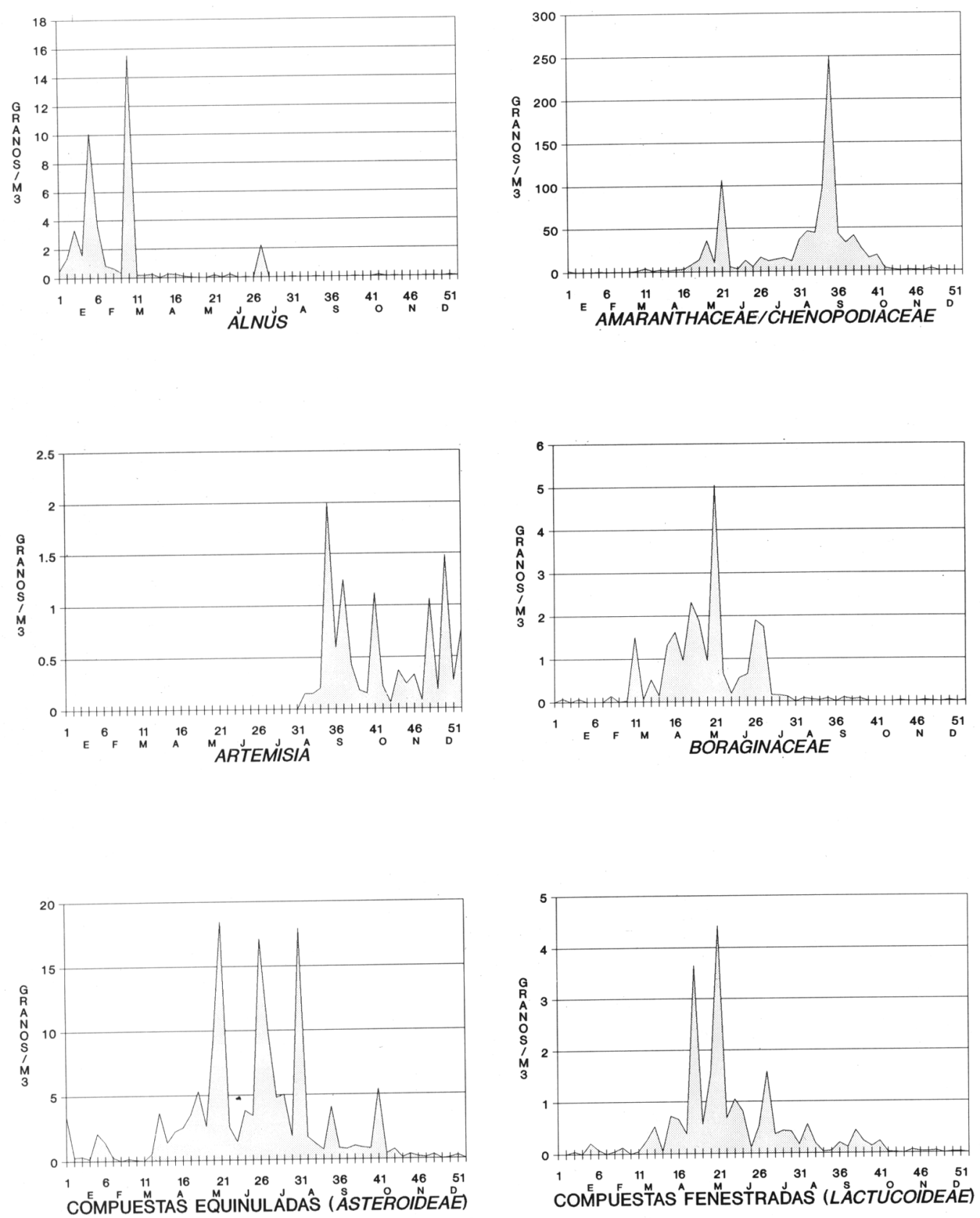

Figura 1. Variación a lo largo del año de las concentraciones semanales expresadas en granos $/ \mathrm{m}^{3} \mathrm{de} l o s$ tipos cuantitativamente más importantes del contenido polínicos de la atmósfera de Huelva. Elaboradas a partir de medias de cuatro años (1989-1992). Change through the year of weekly concentration showed in grains/ $\mathrm{m}^{3}$ of the quantitatively most important kinds of the pollen content in the Huelva atmosphere. The figure it's based upon four years averages (1989-1992). 

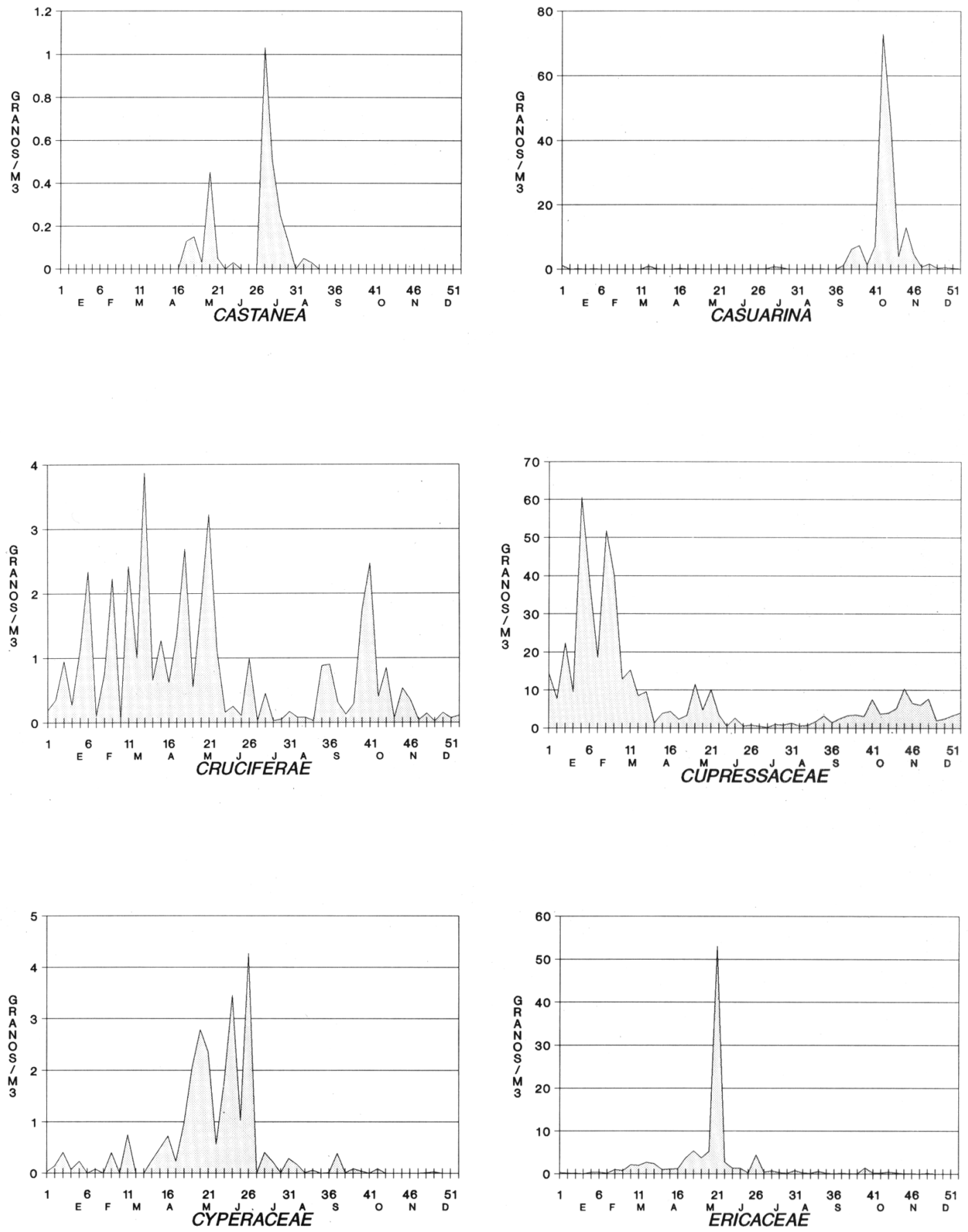

Figura 1. (Continuación). 

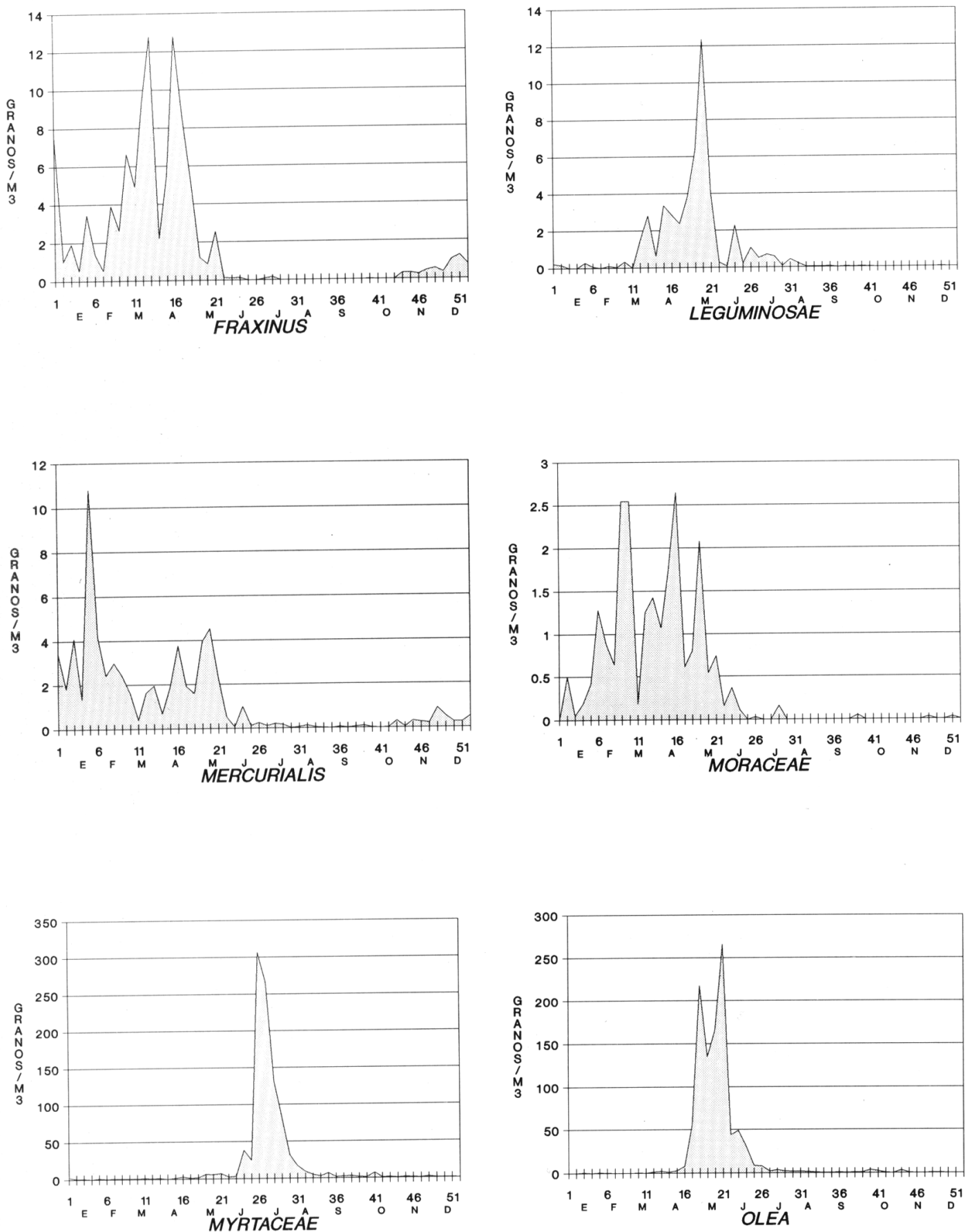

Figura 1. (Continuación). 

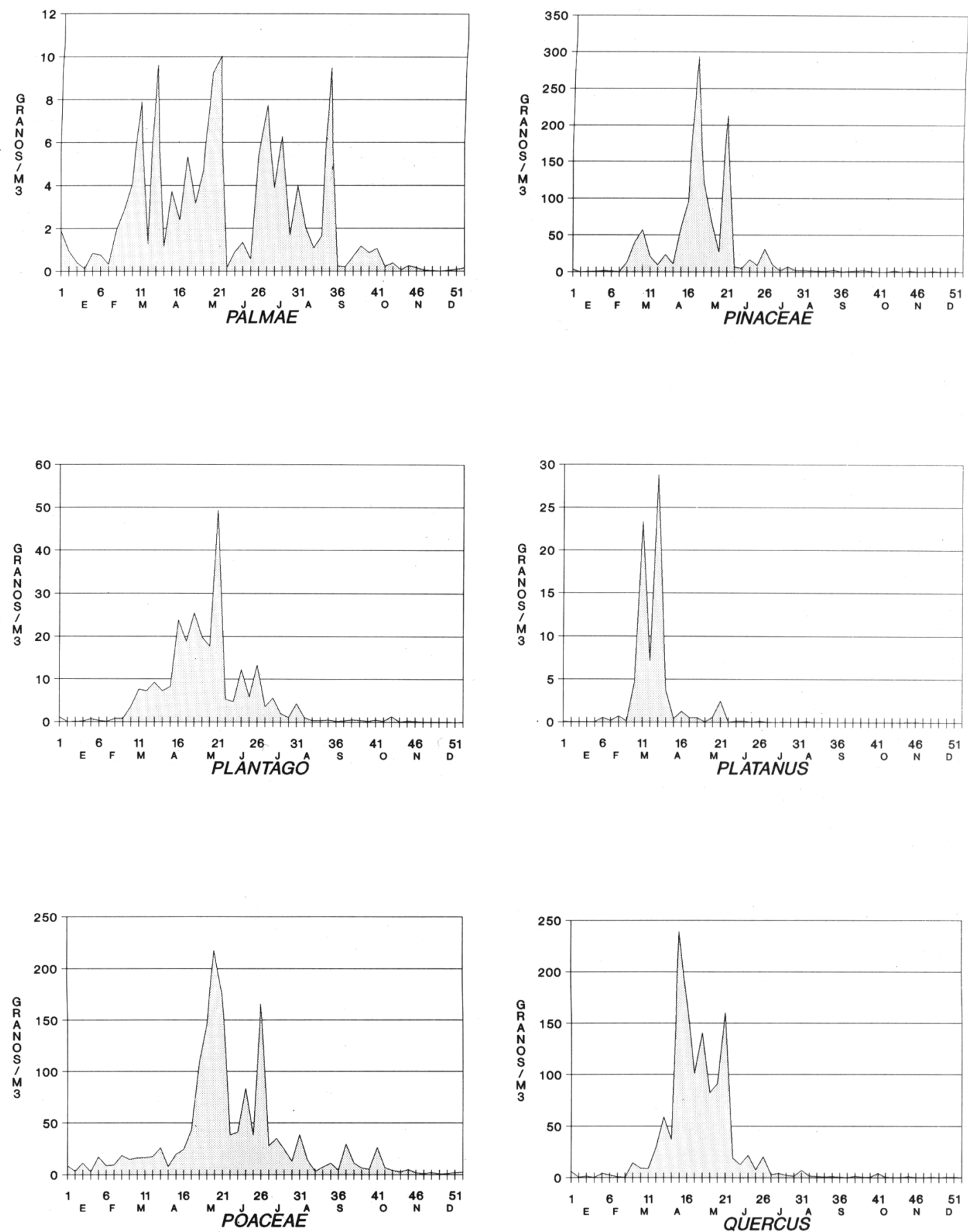

Figura 1. (Continuación). 

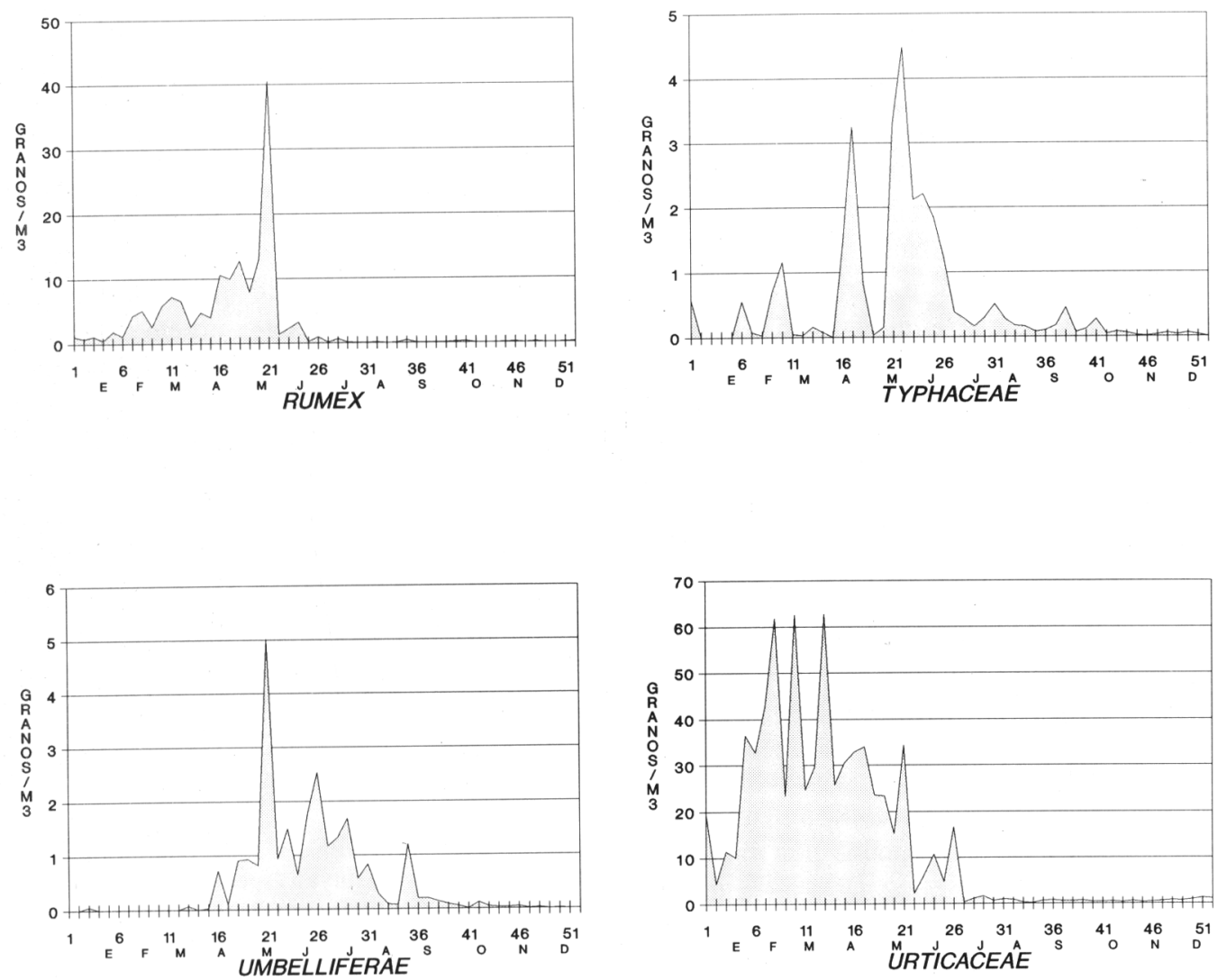

Figura 1. (Continuación).

(4.5 granos $\left./ \mathrm{m}^{3}\right)$, en la 20 la máxima concentración de Poaceae (250 granos $/ \mathrm{m}^{3}$ ) y Leguminosae (12 granos $/ \mathrm{m}^{3}$ ) y en la 21 se miden concentraciones máximas de Olea (250 granos $\left./ \mathrm{m}^{3}\right)$, Plantago $(50$ granos $\left./ \mathrm{m}^{3}\right)$, Ericaceae (50 granos $/ \mathrm{m}^{3}$ ), Rumex (40 granos $\left./ \mathrm{m}^{3}\right)$, Compuestas equinuladas (Asteroideae) (18 granos $/ \mathrm{m}^{3}$ ) y Boraginaceae (5 granos $/ \mathrm{m}^{3}$ ).

En junio existe un descenso tanto de la variedad como de las concentraciones de los palinomorfos señalados en el mes anterior.
Aparecen pequeñas concentraciones de Typhaceae y Cyperaceae y en la última semana del mes se produce la máxima recogida de polen de Myrtaceae (300 granos $/ \mathrm{m}^{3}$ ).

Los meses estivales se caracterizan por una bajada generalizada de las concentraciones polínicas, aunque en julio se siguen recogiendo altas concentraciones de polen Myrtaceae. Durante el verano aparecen picos de polen de Compuestas equinuladas y bajas concentraciones de Castanea, Palmae y Umbelliferae. Entre 
agosto y septiembre predomina el polen de Amaranthaceae/ Chenopodiaceae, alcanzándose 250 granos $/ \mathrm{m}^{3}$ en la semana 35 .

En octubre aparece el polen de Casuarina, que supone casi la totalidad del polen recogido en esa fecha.

En noviembre y diciembre el espectro polínico es pobre, se miden bajas cantidades de polen de Artemisia, Cruciferae y Cupressaceae.

Por último comentamos la aparición en el espectro de algunos tipos entomófilos. Destacan las concentraciones de 20 granos $/ \mathrm{m}^{3}$ de compuestas equinuladas medidas en varias semanas julio y agosto, atribuibles en gran medida al polen de Helianthus que se presenta en el aire producto de fenómenos de reflotación inducidos por las máquinas cosechadoras de girasol. Subrayar también la recogida de polen de Castanea a comienzos de julio, testimonio de transporte polínico a gran distancia desde los castañares más cercanos situados en la sierra a $100 \mathrm{~km}$. del captador.

\section{CONCLUSIONES}

El contenido polínico se caracteriza por la presencia significativa de polen durante nueve meses del año, aunque por tratarse de una localidad costera, expuesta a régimen de brisas diarias y a vientos procedentes del mar, la cantidad polínica total recogida siempre será menor que la establecida en ciudades cercanas situadas en el interior.

El polen de gramíneas domina en el espectro aeropalinológico de Huelva, consecuencia de las características ecológicas, fenológicas y aeropalinológicas de la familia.

A pesar de la tradicional sustitución de la vegetación propia de la zona por especies forestales y cultivos herbáceos y leñosos, el polen procedente de las plantas autóctonas (Quercus, Chenopodiaceae y Cupressaceae) representa un alto porcentaje de los palinomorfos totales.
La vegetación introducida (Olea, Pinaceae, Myrtaceae) aporta gran cantidad de polen al espectro o aumenta la diversidad del mismo (Helianthus, Leguminosae, Castanea).

Las concentraciones polínicas de origen urbano o semiurbano dependen de la proliferación en las ciudades de solares y terrenos sin edificar (caso de Urticaceae) y de la abundancia y diversidad de vegetación ornamental en parques y jardines (caso de Cupressaceae, Casuarina, Moraceae, Palmae, etc.).

Salvo en el caso de Cupressaceae, que incluye polen procedente de la vegetación natural y de representantes ornamentales, el resto de la flora ornamental arbórea de la ciudad (Casuarina, Moraceae, Palmae, Ulmaceae y Ligustrum, etc.) apenas se ve reflejada en el espectro polínico, hecho debido a la escasez de espacios verdes en la ciudad durante los años de estudio y a que los captadores polínicos situados a considerables alturas en el centro de las ciudades, recogen con más facilidad e polen procedente del exterior de las mismas.

\section{ANEXO}

Tipo Alnus: Alnus glutinosa. Tipo Amaranthaceae Chenopodiaceae: incluye a especies de Amaranthus, Arthrocnemum, Atriplex, Beta, Chenopodium, Halimione, Salicornia, Salsola, Sarcocornia y Suaeda. Tipo Artemisia: Artemisia caerulescens, A. crithmifolia y A. absinthium. Tipo Boraginaceae: Echium plantagineum y E.gaditanum. Tipo Casuarina: Casuarina equisetifolia y $C$. cunninghamiana. Tipo Compuestas equinuladas (Asteroideae): es un tipo artificial en el que se incluye el polen equinulado procedente de especies pertenecientes a 27 géneros de esta familia. Tipo Compuestas fenestradas (Lactucoideae): es un tipo artificial en el que se incluye el polen fenestrado procedente de especies pertenecientes a 30 géneros de esta familia. Tipo Cruciferae: incluye a especies de 23 géneros. Tipo Cupressaceae: Cupressus arizonica, C. sempervirens, C. macrocarpa, Juniperus oxycedrus, J. turbinata y especies de Thuja. Tipo Cyperaceae: incluye a 6 especies del género Cyperus y 9 especies del género Carex. Tipo 
Ericaceae: Erica umbellata, E. australis, E. andevalensis, E. arborea, E. ciliaris, Calluna vulgaris y Arbutus unedo. Tipo Fraxinus: Fraxinus angustifolia, F. excelsior, F. ornus, Phyllirea angustifolia y $P$. latifolia Tipo Leguminosae: incluye a especies de los géneros Coronilla y Lotus. Tipo Mercurialis: Mercurialis annua, M. elliptica y M. tomentosa. Tipo Moraceae: Morus alba, M. nigra y Broussonetia papyrifera. Tipo Myrtaceae: Eucalyptus globulus, E. camaldulensis y Myrtus communis. Tipo Olea: Olea europaea. Tipo Palmae: Chamaerops humilis y especies de los géneros Phoenix y Washingtonia. Tipo Pinaceae: Pinus halepensis, $P$. pinaster y $P$. pinea. Tipo Platanus: Platanus hybrida. Tipo Plantago: Plantago major, P. coronopus, $P$. macrorhiza, $P$. serraria, $P$. lanceolata, $P$. lagopus, $P$. albicans, $P$. afra y $P$. bellardii. Tipo Poaceae : incluye a especies de 63 géneros presentes en la flora de Andalucía Occidental. Tipo Quercus: Quercus coccifera, $Q$. rotundifolia, $Q$. suber, $Q$. pyrenaica, $Q$. faginea, $Q$. canariensis y $Q$. lusitanica. Tipo Rumex: Rumex angiocarpus, $R$. tingitanus, $R$. scutatus, $R$. acetosella y $R$. bucephalophorus. Tipo Typhaceae: Typha angustifolia, $T$. dominguensis y $T$. latifolia. Tipo Umbelliferae: incluye a especies de Ammi, Capnophyllum, Daucus, Oenanthe, Peucedanum, Pimpinella, Scandix, Thapsia y Torilis. Tipo Urticaceae: Urtica membranacea, U. urens, Parietaria judaica y P. mauritanica.

\section{BIBLIOGRAFÍA}

ALMARZA MATA, C. -1984- Fichas hídricas normalizadas $y$ otros parámetros hidrometeorológicos. Instituto Nacional de Meteorología. Madrid.

CABEZUDO, B., M. TRIGO, M. RECIO y F.J. TORO -1994- Contenido polínico de la atmósfera de Málaga: años 1992 y 1993. Acta Botánica Malacitana, 19: 137-144.

CANDAU, P. \& F.J. GONZÁLEZ MINERO -1992Relationship between vegetation and pollen spectrum in SW Spain. Isr. J. Bot., 41: 285-295.

CANDAU, P., F.J. GONZÁLEZ MINERO y M.L. GONZÁLEZ ROMANO -1993- Relación entre la flora aeropalinológica y el espectro polínico de tres ciudades del SW de España. Gior. Bot. Italiano, 127 (2): 229-241.

COUR, P. -1974- Nouvelles techniques de détection des flux et des retombées polliniques: étude de la sédimentation des pollens déposés à la surface du sol. Pollen et Spores, 16: 103-141.
COUR, P. \& M. VAN CAMPO - 1980- Prévisions de récoltes à partir du contenu pollinique de l'átmosphère. C. R. Acad. Sc. Paris, 290: 10431046.

DIAZ RUBIO, M. y J. BARRIOS GUTIÉRREZ 1942- La polinosis en Cádiz. Rev. Clín. Esp., 4: 344-348

FERNÁNDEZ, P., C. DIAZ DE LA GUARDIA y F. VALLE -1990- Análisis polínico en la atmósfera de Granada, resultados febrero-junio (19891990). An. Asoc. Palinol. Leng. Esp., 5:29-38.

GALÁN,C. -1987- Catalogación y modelos de variación del polen aerovagante de la ciudad de Córdoba. Tesis Doctoral. Universidad de Córdoba Córdoba.

GONZÁLEZ ROMANO, M.L., P. CANDAU \& F.J. GONZÁLEZ MINERO -1992- Pollen calendar of Seville and its relation to allergies. J. Invest. Allergol. Clin. Inmunol., 2 (6): 323-328.

GONZÁLEZ ROMANO, M.L., P. CANDAU y F.J. GONZÁlEZ MINERO -1993- Estudio aeropalinológico de Sevilla. An. Asoc. Leng. Esp., 6: 39-50.

HURTADO, I. \& P. ALSON -1990- Air pollen dispersal in tropical area. Aerobiologia, 6 (2): 122-127.

KEYNAN, N., Y. WAISEL, A. SHOMER-ILAN, A. GORDEN \& S. BRENER -1991- Annual variations of airborne pollen in the Coastal Plain of Israel. Grana, 30: 447-480.

MUELLENDERS, W., B. PLASMANNE \& M. DIRICKY -1972-La pluie pollinique à Louvainla-Neuve en 1971. Travaux du Laboratoire de Palynologie et de Phytosociologie. Université de Louvain. Louvain.

VALDÉS, B., M.J. DÍEZ e I. FERNÁNDEZ -1987Atlas Polínico de Andalucía Occidental. Instituto de desarrollo regional (Universidad de Sevilla). Excma. Diputación de Cádiz, Sevilla.

Aceptado para su publicación en Junio de 1995

Dirección de los autores. Dpto. Biología Vegetal y Ecología. Universidad de Sevilla. Apdo. 874. 41012 Sevilla. 